\title{
Building the Bridge: Molecular Imaging Biomarkers for 21st Century Cancer Therapies
}

\author{
Mark A. Sellmyer ${ }^{1,2 *}$, Iris K. Lee*1,3, and David A. Mankoff ${ }^{1}$ \\ ${ }^{1}$ Department of Radiology, University of Pennsylvania, Philadelphia, Pennsylvania; ${ }^{2}$ Department of Biochemistry and Biophysics, \\ University of Pennsylvania, Philadelphia, Pennsylvania; and ${ }^{3}$ Department of Bioengineering, University of Pennsylvania, Philadelphia, \\ Pennsylvania
}

\begin{abstract}
Precision medicine, in which the molecular underpinnings of the disease are assessed for tailored therapies, has greatly impacted cancer care. In parallel, a new pillar of therapeutics has emerged with profound success, including immunotherapies such as checkpoint inhibitors and cell-based therapies. Nonetheless, it remains essential to develop paradigms to predict and monitor for therapeutic response. Molecular imaging has the potential to add substantially to all phases of cancer patient care: predictive, companion diagnostics can illuminate therapeutic target density within a tumor, and pharmacodynamic imaging biomarkers can complement traditional modalities to judge a favorable treatment response. This "Focus on Molecular Imaging" article discusses the current role of molecular imaging in oncology and highlights an additional step in the clinical paradigm termed a therapeutic biomarker, which serves to assess whether next-generation drugs reach their target to elicit a favorable clinical response.
\end{abstract}

Key Words: molecular imaging; PET; companion diagnostics; personalized medicine; targeted cancer therapy; cancer biomarkers

J Nucl Med 2021; 62:1672-1676

DOI: 10.2967/jnumed.121.262484

$\mathbf{T}$ here has been rapid progress in the development of targeted cancer therapies over the past 20 years. Cytotoxic chemotherapeutic regimens are still effective and often used but lack specificity and frequently result in significant side effects. One of the goals of the precision medicine era is to better tailor treatments to the individual's particular cancer. This tailoring has evolved to include targeted treatments that are based on biomarkers present in the tumor (1). The therapeutic strategies have diversified in the postgenomic era, taking advantage of biologic agents, recombinant proteins, chimeric approaches, radiotherapies, and even so-called "living drugs" based on engineered cells or viruses that can sense and respond to a particular pathology. As the diversity of approaches has advanced, molecular imaging is uniquely positioned to broaden its critical role in modern therapy development, therapeutic monitoring, and response assessment $(2,3)$.

Molecular diagnostics such as tissue or blood-based biomarkers continue to play an important role and are often a gold standard in

\footnotetext{
Received Apr. 26, 2021; revision accepted Aug. 5, 2021.

For correspondence or reprints, contact Mark A. Sellmyer (mark.sellmyer@ pennmedicine.upenn.edu) and David Mankoff (david.mankoff@pennmedicine. upenn.edu).

${ }^{*}$ Contributed equally to this work.

Published online August 26, 2021.

COPYRIGHT @ 2021 by the Society of Nuclear Medicine and Molecular Imaging.
}

terms of being both predictive and prognostic biomarkers. To ensure that the therapeutic potential of targeted drugs is realized, there has been a push for a drug-diagnostic codevelopment model in which diagnostic tests and drugs are developed in parallel (4). For example, immunohistochemistry for estrogen receptor (ER) expression plays an important role as a biomarker forecasting tumor aggressivity and response to estrogen pathway therapies in breast cancer (5), and molecular characterization of hematologic malignancies such as diffuse large B-cell lymphoma guides the use of modern therapies such as chimeric antigen receptor (CAR) $\mathrm{T}$ cells and bispecific antibodies targeting cell surface markers, such as CD19 and CD20 (6). Other recent examples include peripheral blood sampling for cytokines associated with T-cell activation (e.g., IL-12) and circulating tumor DNA $(7,8)$. These examples highlight how tissue-sampling approaches can complement circulating biomarkers that capture the state of the pathology or therapy in action. However, despite their benefits, all these biomarkers are currently being assessed using in vitro assays with biopsied tissues and blood samples. Directsampling approaches are limited by their invasive nature, which makes repeated sampling impractical. Furthermore, direct sampling comes with the potential trade-off of failing to capture tumor heterogeneity, or assess multifocal disease, and can be prone to sampling errors and artifacts (9). Indeed, even robust gold standard techniques with biologically relevant results can be misleading as to whether a therapy may be successful. For example, programmed-death ligand 1 (PD-L1) expression was not predictive of overall response to immune checkpoint therapy with nivolumab in patients with recurrent metastatic urothelial carcinoma (10). This result highlights that current methods of assessing biomarkers not only are invasive and impractical but also might not yet be powerful enough to accurately predict whether the patient will respond to certain treatments. Thus, key developments in molecular imaging are needed to address the current limitations and to provide clinicians with the information to best tailor cancer therapies.

Here we briefly review the molecular imaging paradigm that has evolved in recent years and consider new ways of applying molecular imaging to predict and assess response to 21 st century cancer therapeutics, including the unique ability of molecular imaging to capture targeted therapy delivery to tumor sites. Concepts such as integrated and integral biomarkers and the evolving use of biomarkers in oncology clinical trials - early response indicators and surrogate endpoints - were discussed in more depth previously (3).

\section{INTRODUCTION ON TYPES OF BIOMARKERS}

We consider a clinical imaging paradigm of informed decision making using several branch points that include predictive 
markers, therapeutic markers, and pharmacodynamic markers (2), guided by definitions used in oncology (11). Predictive markers measure the therapeutic target and ideally give quantitative measures of target expression at the disease site. We define the term therapeutic biomarker as a marker that can measure target engagement or occupancy to guide drug dosing, for example. Pharmacodynamic biomarkers measure biochemical processes or phenotypic outcomes that are downstream from the target to assess whether the drug has had its intended action after treatment. This approach, illustrated in Figure 1, highlights serial branch points in treatment decision making and adds the important strength of molecular imaging markers to assess therapeutic target engagement.

Before discussing each type of biomarker in more depth and with relevant examples, we should note that there are numerous pathologic biomarkers and their partner radiotracers that can be used in different capacities at multiple points in the paradigm. For example, the ER PET imaging described below can be used as both a predictive and a therapeutic biomarker. A key concept in our framework (Fig. 1) is that the timing of imaging dictates the primary capacity in which the biomarker is functioning and providing useful information. In general, a predictive biomarker describes an assessment before treatment. Therapeutic and pharmacodynamic biomarkers are assessed during treatment, typically early after treatment has been applied. Although all markers are in some sense predictive of later response, a distinction is made between markers that predict response in advance of treatment (predictive) and those that require a short exposure to treatment (therapeutic, pharmacodynamic).

\section{PREDICTIVE MARKERS}

A well-known example of a predictive biomarker is PET imaging of ER expression using ${ }^{18} \mathrm{~F}$-fluoroestradiol. ${ }^{18} \mathrm{~F}$-fluoroestradiol uptake has been shown to strongly correlate with ER expression measured by conventional tissue-based assays (12). Clinical studies have demonstrated a robust correlation between baseline ${ }^{18} \mathrm{~F}$-fluoroestradiol uptake levels and therapeutic response, with ${ }^{18} \mathrm{~F}$-fluoroestradiol uptake being highly predictive of breast cancer responsiveness to ER-targeted endocrine therapies and aromatase inhibitors. More importantly, ${ }^{18} \mathrm{~F}$-fluoroestradiol PET has a high negative predictive value, with the lack of uptake strongly suggesting a lack of response, demonstrating how the assessment of ER status with ${ }^{18} \mathrm{~F}$-fluoroestradiol can thus be used to select patients whose tumor expresses the therapeutic target and to guide therapy.

Predictive marker imaging can also be used to guide radionuclide therapy. An example includes the theranostic pairing of ${ }^{68} \mathrm{Ga}-$ DOTATATE (Netspot; Advanced Accelerator Applications) with

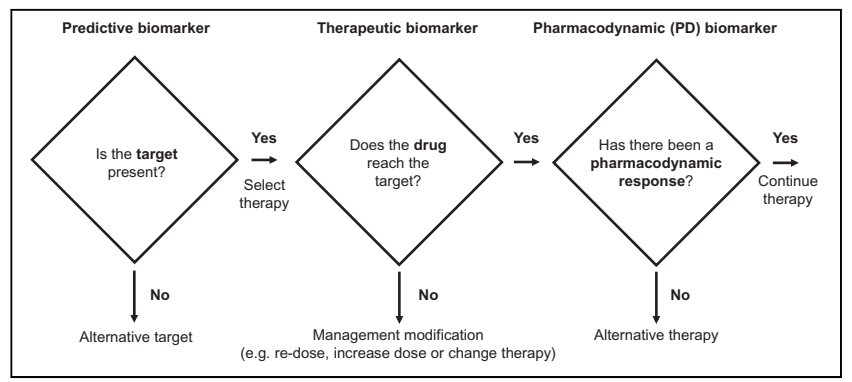

FIGURE 1. Clinical decision pathway incorporating imaging biomarkers. This path highlights 3 different potential roles of molecular imaging as predictive, therapeutic, and pharmacodynamic biomarker. (Adapted from (2).)
${ }^{177}$ Lu-DOTATATE (Lutathera; Advanced Accelerator Applications), a somatostatin-targeted peptide receptor radionuclide therapy for the treatment of neuroendocrine tumors (13). For radionuclide therapy, one can also consider radiopharmaceutical dosimetry, specifically the estimation of radiation dose to normal organs from the radiopharmaceutical, as an important predictive marker for guiding therapy (14). An important area of ongoing dosimetry research is the ability of imaging to assess tumor dose in addition to normalorgan exposure, as a key predictive measure of tumor response. For this task, closely paired diagnostic agents and quantitative tomographic imaging can provide a good estimate of disease targeting to optimize treatment choices and radiopharmaceutical dose selection $(14,15)$, in an elegant use of paired diagnostic-therapeutic radiopharmaceuticals - that is, a theranostic approach.

Although the above examples have been in development for over a decade, many promising predictive markers are in the preclinical stage for emerging therapies. Poly[adenosine diphosphate ribose] polymerase (PARP) 1 has emerged as an attractive anticancer target given its role in DNA damage repair, and the development of PARP inhibitors is on the rise for the treatment of various types of cancers (16). A radiotracer based on the PARP inhibitor AG14699, ${ }^{18} \mathrm{~F}$-fluorthanatrace, is currently at the stage of validation against tissue-based studies for breast and ovarian cancer to assess its predictive value and has the potential to be a clinical predictor of response to PARP inhibitor therapies (17-19).

The use of predictive markers has extended into new classes of therapies to support patient selection and response prediction. The emergence of immunotherapies has motivated the development of antibody-based, immuno-PET probes for imaging therapeutic targets. For example, ${ }^{89} \mathrm{Zr}$-atezolizumab, an anti-PD-L1 antibody, has been developed to assess PD-L1 expression on cancer cells to predict benefit from PD-1/PD-L1 checkpoint blockade therapy (Fig. 2A) (20). Initial results from clinical studies have demonstrated that ${ }^{89} \mathrm{Zr}$-atezolizumab tumor uptake positively correlates to the responsiveness of tumor to PD-L1 blockade therapy with atezolizumab and to both progression-free survival and overall survival. Furthermore, PD-L1 status evaluated by PET imaging has been shown to better predict clinical response than can immunohistochemistry or RNA-sequencing-based biomarkers (20). Imaging of checkpoint protein receptor cytotoxic T-lymphocyte-associated antigen 4 (CTLA4) with ${ }^{89} \mathrm{Zr}$-ipilimumab is currently being studied in a clinical trial setting (NCT03313323) to determine the correlation between tumor uptake of radiolabeled ipilimumab and response to ipilimumab therapy.

To date, tumor-specific and therapy-specific markers have been the dominant classes of predictive PET imaging biomarkers. A deviation in this trend is noted with the emergence of PET tracers developed to image pan-tumor markers, such as fibroblast activation protein (FAP), in the tumor microenvironment (21). FAPspecific enzyme inhibitor has been radiolabeled with ${ }^{68} \mathrm{Ga}$ to image FAP-positive reactive stromal content in various solid tumors with high contrast $\left({ }^{68} \mathrm{Ga}-\mathrm{FAP}\right.$ inhibitor) (22). The DOTAcoupled, chelated nature of the radiotracer highlights that the radioisotope can easily be switched with a therapeutic isotope such as ${ }^{177} \mathrm{Lu}$, enabling potential theranostic pairing of ${ }^{68} \mathrm{Ga}-\mathrm{FAP}$ inhibitor imaging with ${ }^{177} \mathrm{Lu}-\mathrm{FAP}$ inhibitor therapy. Given its selective expression in tumor stromal cells, FAP has also emerged as a promising stromal cell target for solid tumor immunotherapy, including CAR T-cell therapy, portending the use of FAP PET imaging as a companion diagnostic for FAP CAR T-cell therapy to assess the biodistribution of the target (23). 


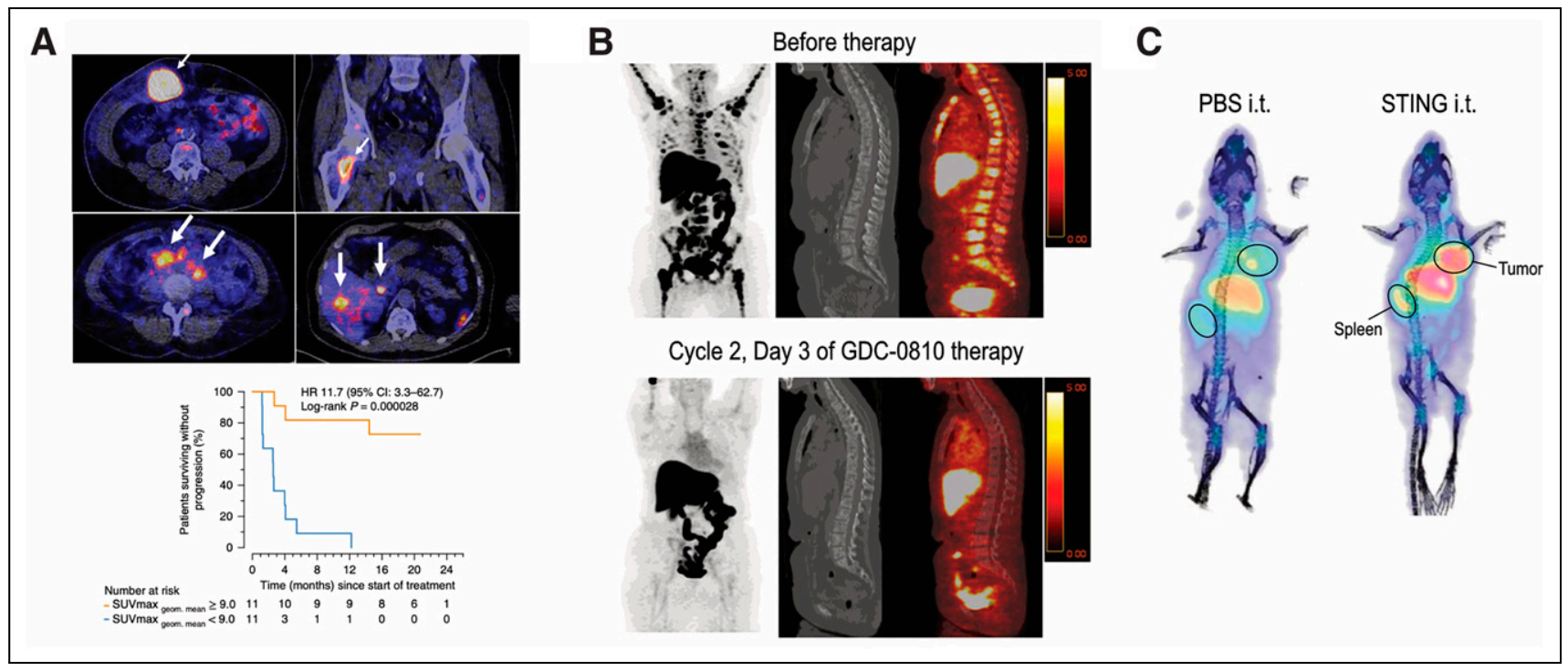

FIGURE 2. Examples of predictive (A), therapeutic (B), and pharmacodynamic (C) markers for 21 st century therapies. (A) ${ }^{89} \mathrm{Zr}$-atezolizumab tumor uptake (arrows) predicts clinical response to PD-L1 blockade therapy and overall progression-free survival. (Adapted with permission of $(20)$.) $(\mathrm{B}){ }^{18} \mathrm{~F}-\mathrm{flu}-$ oroestradiol PET/CT images demonstrate complete suppression of $\mathrm{O}-\left(2-{ }^{18} \mathrm{~F}\right.$-fluoroethyl)-L-tyrosine-avid lesions with ER-targeting therapeutic GDC-0810, highlighting potential role of ${ }^{18} \mathrm{~F}$-fluoroestradiol PET/CT as biomarker of ER occupancy or downregulation for determining dosages of various ER-targeted therapeutics. (Adapted with permission of (25).) (C) Immuno-PET imaging of ${ }^{89} \mathrm{Zr}$-desferrioxamine-ICOS on day 2 after tracer administration shows increased uptake of tracer by ICOS-positive activated T cells in tumor, tumor-draining lymph nodes, and spleen of mouse treated with stimulatorof-interferon-genes protein (STING) i.t. compared with phosphate-buffered saline (PBS) i.t. (Reprinted with permission of $(43)$.) HR = hazard ratio; i.t. = intratumoral.

\section{THERAPEUTIC BIOMARKERS}

Therapeutic biomarkers, which are focused on assessing whether the therapy has reached the target, are a rapidly evolving area in molecular imaging. Traditional examples include diagnostic and therapeutic radiopharmaceuticals (e.g., neuroendocrine tumor agents), including therapeutic agents that intrinsically emit a measurable signal, such as ${ }^{131} \mathrm{I}$ therapy (24). Dosimetry with ${ }^{131} \mathrm{I}$ allows optimal dosing in refractory thyroid cancers, yielding measurements that optimize the radiotherapy dose to the target without surpassing toxicity limits to key organs such as the lungs and the bone marrow. With the advent of quantitative molecular imaging biomarkers, imaging can also assess drug dosing for standard therapeutics. An initial study performed to measure ER availability with ${ }^{18} \mathrm{~F}$-fluoroestradiol before and during fulvestrant therapy demonstrated that residual ${ }^{18} \mathrm{~F}$-fluoroestradiol uptake after treatment is associated with early clinical disease progression (12). This observation motivated the use of ${ }^{18} \mathrm{~F}$-fluoroestradiol to measure target engagement of selective ER degrader drugs for determining the optimal dose to achieve clinically significant ER inhibition (Fig. 2B) and is now increasingly applied in the development of new selective ER degrader agents (25).

The expansion and translation of imaging-based therapeutic monitoring may be even more critical for 21 st century therapies, including immunotherapies and so-called living drugs that entail gene and cell therapies. Living drugs are uniquely challenging to monitor because of their dynamic behavior in vivo. For example, a cell therapy may undergo autonomous regulation, with dynamic expansion on target recognition, which includes orders-of-magnitude increases in the number of therapeutic cells and related contraction in cell number after target clearance. This type of therapy does not follow the traditional pharmacokinetics principles developed around conventional chemotherapeutic and biologic drugs
(26). Furthermore, the dynamic and self-regulating underpinnings of a living-drug approach magnify the concern about on-target, off-tumor toxicities, such as a case of pulmonary toxicity and subsequent death in a CAR T-cell-treated patient, thought to be related to the native expression of ERBB2 on normal lung tissue (27). Therefore, developing therapeutic biomarkers for cell-based therapies to better understand the dose and dynamic behaviors of the living drugs is a key milestone.

There are 2 main strategies for tracking living drugs over time: direct and indirect labeling (28). For direct labeling methods, cells are labeled with imaging agents such as ${ }^{89} \mathrm{Zr}$-oxine and monitored over a time course of hours to days. Weist et al. recently demonstrated ${ }^{89} \mathrm{Zr}$-oxine labeling of interleukin 13 receptor $\alpha 2$-targeted and prostate stem cell antigen-targeted CAR $\mathrm{T}$ cells to monitor in vivo trafficking to glioblastoma tumors and subcutaneous prostate tumors, respectively (29). The oxine-labeled CAR T cells were detectable for at least $6 \mathrm{~d}$ after labeling, and the labeling did not result in a significant reduction in functionality of the CAR T cells.

Although relatively straightforward and inexpensive, direct labeling strategies are hampered by dilution of signal on cell division and death and is therefore not amenable to medium- or long-term monitoring of therapy (28). Two alternative approaches include using cell surface labels (e.g., inducible T-cell costimulator [ICOS] or CD8) coupled with immuno-PET radioprobes or reporter gene imaging platforms. In the latter, living drugs such as CAR T cells are transduced with a reporter gene of interest that can be specifically targeted via a complementary radioprobe, allowing for imaging over the entire lifetime of the cell, with faithful signal amplification with each cell division (28). One of the most extensively studied PET reporter genes is HSV1-tk. HSV1-tk-engineered cytotoxic $\mathrm{T}$ lymphocytes expressing interleukin-13 CAR to target human gliomas after surgical resection were monitored using a radiolabeled analog of the anti-herpes drug penciclovir, ${ }^{18} \mathrm{~F}-\mathrm{FHBG}$ 
(9-(4- ${ }^{18}$ F-fluoro-3-[hydroxymethyl]butyl)guanine). PET imaging of ${ }^{18} \mathrm{~F}-\mathrm{FHBG}$ demonstrated cytotoxic T-lymphocyte accumulation in the areas of the tumor, noting that ${ }^{18} \mathrm{~F}$-FHBG does not naturally cross the intact blood-brain barrier (30).

FOLH1 encodes for prostate-specific membrane antigen and is an example of a PET reporter gene that has gained a lot of attention, given its human origin. It has been engineered into CD19 CAR T cells to assess its trafficking to CD19-expressing tumor cells in mice, with high sensitivity (31). An elegant finding from this work was the demonstration that the number of intratumoral CAR T cells derived from the PET images did not correlate with the T-cell counts in the blood, suggesting that the peripheral blood may not reflect the degree to which tumors are infiltrated with CAR T cells, which is therapeutically relevant information.

We recently described $E$. coli dihydrofolate reductase and radiotracer derivatives of its highly specific small-molecule inhibitor trimethoprim as a promising reporter gene-probe pair for monitoring CAR $\mathrm{T}$ cells. ${ }^{18} \mathrm{~F}$-trimethoprim imaging showed early residence of $E$. coli dihydrofolate reductase-expressing disialoganglioside 2-targeted CAR T cells in the spleen by day 7, followed by on-target accumulation in disialoganglioside 2-positive tumor by day 13 (32). Ex vivo anti-human CD8 immunohistochemistry showed that as few as 11,000 CD8 E. coli dihydrofolate reductase-expressing CAR T cells per cubic millimeter of tumor tissue could be detected in the PET images.

Beyond CAR T cells, approaches using reporter gene imaging have been established as a common platform to monitor other $21 \mathrm{st}$ century therapies. Notably, gene therapy/gene replacement with adeno-associated virus, and cancer therapies using oncolytic viruses, are important fields in which the penetrance and durability of the viral vector often have an impact on the therapeutic outcome $(33,34)$.

\section{PHARMACODYNAMIC BIOMARKERS}

As the above paradigms are geared for making proper clinical management choices from the beginning of a therapeutic intervention (predictive) and measuring whether the drug reaches its target (therapeutic), crucial downstream measures of efficacy can be assessed with pharmacodynamic biomarkers. These markers are well known to the nuclear medicine community and have been studied for decades. Two key classes of pharmacodynamic markers are metabolic and proliferative measures. The most widely adopted metabolic radiotracer is ${ }^{18} \mathrm{~F}-\mathrm{FDG}$, which functions as a measure of glycolysis (35). Many clinical patient streams rely on PET/CT imaging with ${ }^{18} \mathrm{~F}-\mathrm{FDG}$ for diagnosis, including lymphoma, head and neck tumors, high-risk skin cancer (e.g., melanoma), and breast cancer (36). These patients are treated with diverse chemotherapeutic, biologic, immunotherapeutic, and now cell therapy approaches, and ${ }^{18} \mathrm{~F}$-FDG remains a crucial tool to understand the glycolytic response. Not only have National Comprehensive Cancer Network guidelines been developed to include PET/CT with input from referring clinicians, but also routine response criteria such as PERCIST have been developed to standardize results communication (37). On the horizon, metabolic biomarkers such as amino acid derivatives related to acetate and glutamine are in development. These may be applied in certain clinical situations as surrogates for understanding specific intracellular processes such as fatty acid synthesis or transport of amino acids, particularly for tumors that are not ${ }^{18} \mathrm{~F}-\mathrm{FDG}$-avid and for metabolically targeted therapies $(38,39)$.
Alternatively, a therapy may impact the phenotype of the disease process, a feature for which quantitative molecular imaging is ideally suited. An exciting recent example of imaging a pharmacodynamic response to an estrogen challenge was illustrated by Dehdashti et al. (40). This approach leverages the observation that stimulation of pathways downstream from the ER in breast cancer leads to increased expression of the closely related progesterone receptor. The investigators showed that an increase in uptake of the progesterone receptor radiotracer ${ }^{18} \mathrm{~F}$-fluorofuranylnorprogesterone in response to an estradiol challenge is a potent way to assess for ER receptor function and can predict breast cancer response to endocrine therapy. Pharmacodynamic biomarkers for biologics and living drugs also have great potential, especially with respect to immunooncology.

The focus of imaging pharmacodynamic markers is no longer solely on the tumor itself but rather on the immune system. For example, CD8 minibodies image immune cells in inflammatory conditions and cancer and can be used in conjunction with a baseline image to understand how the immune system responds to immunotherapy such as a checkpoint inhibitor (anti-CTLA4 or antiPD1) (41). Another notable example that is nearing clinical translation is the use of ICOS to monitor "stimulator-of-interferon-genes" protein agonist immune activation or CAR T-cell trafficking $(42,43)$. Xiao et al. (43) showed that immuno-PET imaging of ICOS with ${ }^{89} \mathrm{Zr}$-desferrioxamine-ICOS enabled specific detection of activated $T$ cells and their coordinated immune response to stimulator-of-interferon-genes protein and programmed cell death protein 1 checkpoint blockade in the setting of Lewis lung cancer models, highlighting the promising potential of ICOS imaging as a way to monitor T-cell-mediated immune response to various immunotherapies (Fig. 2C). Xiao et al. (43) also demonstrated that the ICOS signal is detectable before changes in tumor volume, suggesting that ICOS imaging will allow for highly sensitive, early detection of response, compared with traditional anatomic imaging approaches.

Despite the success of biologic therapies and antibodies (including bispecifics), integration of similar such protein-based imaging tools into the clinical paradigm has been challenging, in part because of the long circulation time of many of these therapies themselves and the practical challenges of imaging full-length antibodies, for example. For 21 st century therapy imaging, small molecules or immune molecules engineered specifically for imaging will continue to play a large role, and efforts to make smaller biologic probes using radioisotopes with shorter half-lives have shown promise (41).

\section{CONCLUSION}

The use of imaging biomarkers, in their diverse capacities, can impact and improve on a one-size-fits-all approach to medical diagnosis and treatment. Precision medicine promises that with a deep understanding of the molecular mechanisms and pathology heterogeneity, tailored therapies can be prescribed for the improved treatment and health of patients. This molecular imaging biomarker paradigm for both cancer clinical trials and future clinical applications serves as a reference for basic scientific developments in the field of cancer molecular imaging and a formulaic approach to guide clinical trials. Imaging serves to complement diagnostics based on in vitro assays and tissue sampling, especially in terms of predictive biomarkers. Optimizing the dose regimen and understanding whether the drug reaches the pathology is a key component of therapeutic biomarkers, and finally, pharmacodynamic biomarkers are used to assess the 
downstream processes that are affected by the drug and ultimately entail the tumor response to therapy.

The future outlook for imaging biomarkers continues to be bright. As there has been an acceleration in the development of living drugs and new 21 st century therapeutics, the field of molecular imaging should be positioned to meet the needs of pharmaceutical development efforts in terms of companion diagnostics and therapeutic biomarker drug assessment, with an eye toward clinical applications and integration.

\section{DISCLOSURE}

Mark Sellmyer is an intellectual property inventor and equity holder in Vellum Biosciences, a company supporting the commercialization of trimethoprim radiotracers. David Mankoff is an inventor on trimethoprim radiotracers and also has a financial conflict of interest related to licensing of a PET agent for PARP imaging. Mark Sellmyer is supported by the Burroughs Wellcome Fund Career Award for Medical Scientists and the NIH Office of the Director Early Independence Award (DP5-OD26386). David Mankoff received support from Susan G Komen grant SAC130060 and NIH grants R01CA211337 and R33-CA225310 relevant to this article. No other potential conflict of interest relevant to this article was reported.

\section{REFERENCES}

1. Moscow JA, Fojo T, Schilsky RL. The evidence framework for precision cancer medicine. Nat Rev Clin Oncol. 2018;15:183-192.

2. Mankoff DA, Edmonds CE, Farwell MD, Pryma DA. Development of companion diagnostics. Semin Nucl Med. 2016;46:47-56.

3. Mankoff DA, Pryma DA, Clark AS. Molecular imaging biomarkers for oncology clinical trials. J Nucl Med. 2014;55:525-528.

4. Jørgensen JT. Companion and Complementary Diagnostics: From Biomarker Discovery to Clinical Implementation. Academic Press; 2019:2-46.

5. Hammond ME, Hayes DF, Dowsett M, et al. American Society of Clinical Oncology/College of American Pathologists guideline recommendations for immunohistochemical testing of estrogen and progesterone receptors in breast cancer. J Clin Oncol. 2010;28:2784-2795.

6. Sehn LH, Salles G. Diffuse large B-cell lymphoma. N Engl J Med. 2021;384: $842-858$.

7. Dawson SJ, Tsui DW, Murtaza M, et al. Analysis of circulating tumor DNA to monitor metastatic breast cancer. N Engl J Med. 2013;368:1199-1209.

8. Nixon AB, et al. Peripheral immune-based biomarkers in cancer immunotherapy: can we realize their predictive potential? J Immunother Cancer. 2019;7:325.

9. Marciscano AE, Thorek DLJ. Role of noninvasive molecular imaging in determining response. Adv Radiat Oncol. 2018;3:534-547.

10. Sharma $\mathrm{P}$, Callahan MK, Bono $\mathrm{P}$, et al. Nivolumab monotherapy in recurrent metastatic urothelial carcinoma (CheckMate 032): a multicentre, open-label, two-stage, multi-arm, phase 1/2 trial. Lancet Oncol. 2016;17:1590-1598.

11. Henry NL, Hayes DF. Uses and abuses of tumor markers in the diagnosis, monitoring, and treatment of primary and metastatic breast cancer. Oncologist. 2006;11: 541-552.

12. Fowler AM, Clark AS, Katzenellenbogen JA, Linden HM, Dehdashti F. Imaging diagnostic and therapeutic targets: steroid receptors in breast cancer. $\mathrm{J} \mathrm{Nucl} \mathrm{Med}$. 2016;57(suppl 1):75S-80S.

13. Burkett BJ, Dundar A, Young JR, et al. How we do it: a multidisciplinary approach to ${ }^{177}$ Lu DOTATATE peptide receptor radionuclide therapy. Radiology. 2021;298: 261-274.

14. Sgouros G, Bodei L, McDevitt MR, Nedrow JR. Radiopharmaceutical therapy in cancer: clinical advances and challenges. Nat Rev Drug Discov. 2020;19:589-608.

15. Eberlein U, Cremonesi M, Lassmann M. Individualized dosimetry for theranostics: necessary, nice to have, or counterproductive? J Nucl Med. 2017;58 (suppl):97S-103S.

16. Basu B, Sandhu SK, de Bono JS. PARP inhibitors: mechanism of action and their potential role in the prevention and treatment of cancer. Drugs. 2012;72: 1579-1590.
17. Makvandi M, Pantel A, Schwartz L, et al. A PET imaging agent for evaluating PARP-1 expression in ovarian cancer. $J$ Clin Invest. 2018;128:2116-2126.

18. McDonald ES, Doot RK, Pantel AR, et al. Positron emission tomography imaging of poly-(adenosine diphosphate-ribose) polymerase 1 expression in breast cancer: a nonrandomized clinical trial. JAMA Oncol. 2020;6:921-923.

19. Michel LS, et al. PET of poly (ADP-ribose) polymerase activity in cancer: preclinical assessment and first in-human studies. Radiology. 2017;282:453-463.

20. Bensch F, van der Veen EL, Lub-de Hooge MN, et al. ${ }^{89} \mathrm{Zr}$-atezolizumab imaging as a non-invasive approach to assess clinical response to PD-L1 blockade in cancer. Nat Med. 2018;24:1852-1858.

21. Puré E, Lo A. Can targeting stroma pave the way to enhanced antitumor immunity and immunotherapy of solid tumors? Cancer Immunol Res. 2016;4:269-278.

22. Loktev A, Lindner T, Mier W, et al. A tumor-imaging method targeting cancerassociated fibroblasts. $J$ Nucl Med. 2018;59:1423-1429.

23. Lo A, Wang LS, Scholler J, et al. Tumor-promoting desmoplasia is disrupted by depleting FAP-expressing stromal cells. Cancer Res. 2015;75:2800-2810.

24. Pryma DA, Mandel SJ. Radioiodine therapy for thyroid cancer in the era of risk stratification and alternative targeted therapies. J Nucl Med. 2014;55: 1485-1491.

25. Wang Y, Ayres KL, Goldman DA, et al. ${ }^{18} \mathrm{~F}$-fluoroestradiol PET/CT measurement of estrogen receptor suppression during a phase I trial of the novel estrogen receptor-targeted therapeutic GDC-0810: using an imaging biomarker to guide drug dosage in subsequent trials. Clin Cancer Res. 2017;23:3053-3060.

26. Mueller KT, Maude SL, Porter DL, et al. Cellular kinetics of CTL019 in relapsed/ refractory B-cell acute lymphoblastic leukemia and chronic lymphocytic leukemia. Blood. 2017;130:2317-2325.

27. Brudno JN, Kochenderfer JN. Toxicities of chimeric antigen receptor T cells: recognition and management. Blood. 2016;127:3321-3330.

28. Ashmore-Harris C, Iafrate M, Saleem A, Fruhwirth GO. Non-invasive reporter gene imaging of cell therapies, including T cells and stem cells. Mol Ther. 2020; 28:1392-1416.

29. Weist MR, Starr R, Aguilar B, et al. PET of adoptively transferred chimeric antigen receptor T cells with ${ }^{89} \mathrm{Zr}$-oxine. J Nucl Med. 2018;59:1531-1537.

30. Keu KV, Witney TH, Yaghoubi S, et al. Reporter gene imaging of targeted T cell immunotherapy in recurrent glioma. Sci Transl Med. 2017;9:eaag2196

31. Minn I, Huss DJ, Ahn HH, et al. Imaging CAR T cell therapy with PSMA-targeted positron emission tomography. Sci Adv. 2019;5:eaaw5096.

32. Sellmyer MA, Richman SA, Lohith $\mathrm{K}$, et al. Imaging CAR T cell trafficking with eDHFR as a PET reporter gene. Mol Ther. 2020;28:42-51.

33. Haywood T, Beinat C, Gowrishankar G, et al. Positron emission tomography reporter gene strategy for use in the central nervous system. Proc Natl Acad Sci USA. 2019;116:11402-11407.

34. Miller A, Suksanpaisan L, Naik S, et al. Reporter gene imaging identifies intratumoral infection voids as a critical barrier to systemic oncolytic virus efficacy. $\mathrm{Mol}$ Ther Oncolytics. 2014;1:14005.

35. Farwell MD, Pryma DA, Mankoff DA. PET/CT imaging in cancer: current applications and future directions. Cancer. 2014;120:3433-3445.

36. Kelloff GJ, et al. Progress and promise of FDG-PET imaging for cancer patient management and oncologic drug development. Clin Cancer Res. 2005;11:27852808.

37. Wahl RL, Jacene H, Kasamon Y, Lodge MA. From RECIST to PERCIST: Evolving Considerations for PET response criteria in solid tumors. J Nucl Med. 2009; 50(suppl 1):122S-150S

38. Kelloff GJ, Hoffman JM, Johnson B, et al. Late imaging with $\left[1-{ }^{11} \mathrm{C}\right]$ acetate improves detection of tumor fatty acid synthesis with PET. J Nucl Med. 2014;55: 1144-1149.

39. Venneti S, Dunphy MP, Zhang H, et al. Glutamine-based PET imaging facilitates enhanced metabolic evaluation of gliomas in vivo. Sci Transl Med. 2015;7: $274 \mathrm{ra} 17$.

40. Dehdashti F, Wu N, Ma CX, Naughton MJ, Katzenellenbogen JA, Siegel BA. Association of PET-based estradiol-challenge test for breast cancer progesterone receptors with response to endocrine therapy. Nat Commun. 2021;12:733.

41. Pandit-Taskar N, Postow MA, Hellmann MD, et al. First-in-humans imaging with ${ }^{89} \mathrm{Zr}$-Df-IAB22M2C anti-CD8 minibody in patients with solid malignancies: preliminary pharmacokinetics, biodistribution, and lesion targeting. J Nucl Med. 2020; 61:512-519.

42. Simonetta F, Alam IS, Lohmeyer JK, et al. Molecular Imaging of chimeric antigen receptor T cells by ICOS-IMMUNOPET. Clin Cancer Res. 2021;27:1058-1068.

43. Xiao Z, Mayer AT, Nobashi TW, Gambhir SS. ICOS is an indicator of Tcell-mediated response to cancer immunotherapy. Cancer Res. 2020;80:30233032 . 